\title{
GEODESIC CONGRUENCES AND INHOMOGENEOUS HEISENBERG FERROMAGNET
}

\author{
Antoni SYM ${ }^{1}$ and Willem WESSELIUS \\ Department of Applied Mathematics, Twente University of Technology, \\ P.O. Box 217, 7500 AE Enschede, The Netherlands
}

Received 7 October 1986; accepted for publication 10 December 1986

\begin{abstract}
A geometric method to find exact solutions to the one-dimensional classical non-homogeneous Heisenberg ferromagnet model is proposed. By constructing a one-parameter family of geodesics on an arbitrary surface in $\mathrm{E}^{3}$ we are able to find both the coupling function of the model and the particular solution to this model.
\end{abstract}

In this paper we present and apply some geometric method to solve the so-called one-dimensional classical non-homogeneous Heisenberg ferromagnet (1DCNHF) model [1];

$S_{, 2}=f S \times S_{, 11}+f_{, 1} S \times S_{, 1}$,

where $S=S\left(x^{1}, x^{2}\right) \in \mathrm{E}^{3}$ is a unit vector spin variable, $f=f\left(x^{1}, x^{2}\right) \in \mathbb{R}$ is a coupling function, $x^{1}\left(x^{2}\right)$ are space (time) independent variables and, finally, comma means differentiation while cross denotes the skew product in $\mathrm{E}^{3}$. Eq. (1) can be used as a model of some interesting physical phenomena [1].

Lakshmanan and Bullough [2] in the case of $f$ linear in $x^{1}$ and Balakrishnan [1] in the case of an arbitrary $f$ have shown eq. (1) can be reduced to the socalled non-homogeneous non-linear Schrödinger equation (NNSE):

$$
\begin{aligned}
& \mathrm{i} q_{, 2}+(f q)_{, 11}+2 f q|q|^{2} \\
& \quad+2 q \int^{x 1} f_{, 1}\left(s, x^{2}\right)\left|q\left(s, x^{2}\right)\right|^{2} \mathrm{~d} s=0,
\end{aligned}
$$

where $q=q\left(x^{1}, x^{2}\right) \in \mathbb{C}$.

Recently some controversial statements on the integrability of eq. (2) (in the sense of soliton theory) have been formulated $[3,4]$. Presumably, the most general function $f$ which admits the integrability of eq. (2) is $f\left(x^{1}, x^{2}\right)=a\left(x^{2}\right) x^{1}+b\left(x^{2}\right)$ (with $a$

\footnotetext{
I Permanent address: Institute of Theoretical Physics, Warsaw University, ul. Hoża 69, 00-681 Warsaw, Poland.
}

and $b$ arbitrary functions of $x^{2}$ ) [4]. In this case eq. (2) is a slight extension of the integrable equation introduced by Calogero and Degasperis [5].

However, we would like to point out that the questionable integrability of eq. (2) is not the main reason why the method of solving of the $1 \mathrm{D}-\mathrm{CNHF}$ model (1) by its reduction to the NNSE (2) seems to be an inefficient one. It turns out that from the computational point of view it is a very difficult task to reconstruct $S$-solutions from the knowledge of $q$ solutions. In order to compute an $S$-solution one has to solve an auxiliary problem: to reconstruct an $E^{3}$ curve from the knowledge of its curvature and torsion which are given in terms of the $q$-solution. In the case $f=1$ (ordinary non-linear Schrödinger equation) this problem is discussed in ref. [6].

This paper provides a novel geometric approach to both eqs. (1) and (2). It is based on ideas and results of the framework of soliton surfaces [7]. The geometry of soliton surfaces for a given soliton system constitutes a spectral extension of the geometry of pseudo-spherical surfaces which is a geometry of the sine-Gordon equation as well [8]. In particular one can show that the parametric lines $x^{2}=$ const on an arbitrary soliton surface of the nonlinear Schrödinger equation (eq. (2) with $f=1$ ) are geodesic lines $[9,10]$. This observation admits the following extension (for the geometric terminology used below see ref. [7] or ref. [11]):

Consider an arbitrary smooth surface $\sum$ in $E^{3}$ 
equipped with local coordinates (called semi-geodesic coordinates) $\left(x^{1}, x^{2}\right)$ distinguished by: (1) parametric lines $x^{2}=$ const are geodesics and (2) along any geodesic $x^{2}=$ const the coordinate $x^{1}=$ arc length parameter. In other words, the metric in $\left(x^{\prime}\right.$, $x^{2}$ ) variables reads

$\mathrm{d} s^{2}=\left(\mathrm{d} x^{1}\right)^{2}+g_{22}\left(x^{1}, x^{2}\right)\left(\mathrm{d} x^{2}\right)^{2}$.

We do not make any specific assumption about the second fundamental form of the surface $\Sigma$ :

$$
\begin{aligned}
\mathrm{II} & =b_{11}\left(x^{1}, x^{2}\right)\left(\mathrm{d} x^{1}\right)^{2}+2 b_{12}\left(x^{1}, x^{2}\right) \mathrm{d} x^{1} \mathrm{~d} x^{2} \\
& +b_{22}\left(x^{1}, x^{2}\right)\left(\mathrm{d} x^{2}\right)^{2} .
\end{aligned}
$$

We assume, however, $b_{11}\left(x^{1}, x^{2}\right) \neq 0$ everywhere on the surface $\Sigma$. We introduce a real function

$f\left(x^{1}, x^{2}\right)=-\sqrt{g_{22}\left(x^{1}, x^{2}\right) /} b_{11}\left(x^{1}, x^{2}\right)$,

and a complex function

$$
\begin{aligned}
& q\left(x^{1}, x^{2}\right)=\frac{1}{2} b_{11}\left(x^{1}, x^{2}\right) \\
& \quad \times \exp \left(\mathrm{i} \int \frac{b_{12}\left(s, x^{2}\right)}{\sqrt{g_{22}\left(s, x^{2}\right)}} \mathrm{d} s\right) .
\end{aligned}
$$

The following geometric objects on $\sum$ are distinguished: fundamental forms (3) and (4), the geodesic congruence $x^{2}=$ const and the field of tangent vectors to $\sum: S=r_{, 1}\left(r=r\left(x^{1}, x^{2}\right)\right.$ is the position vector to the surface $\Sigma$ ). These geometric objects satisfy their own "equations of motion" which can be described as follows.

(1) All the coefficients of the fundamental forms (3) and (4) can be expressed in terms of $f$ - and $q$ functions exclusively while the so-called Gauss-Mainardi-Codazzi equations of the theory of surfaces are reducible to eq. (2).

(2) The kinematics of the geodesic line $x^{2}=$ const is given by

$r_{, 2}=f r_{.1} \times r_{.11}$.

(3) The kinematics of the vector $S=r_{1}$ is given by the $1 \mathrm{D}-\mathrm{CNHF}$ equation (1).

These results enable one to formulate the following algorithm which allows one to find simultaneously (a) a function $f\left(x^{1}, x^{2}\right)$ that is a particular model (1) and (b) a special solution $S\left(x^{1}, x^{2}\right)$ to this model.
(A) Select any smooth surface $\sum$ in $E^{3}$. Refer it to arbitrary (possibly convenient) local coordinates, say, $u$ and $v$.

(B) Working with $(u, v)$ coordinates try to integrate equations for geodesics. The resulting general integral can be cast into the form

$u=v(\sigma ; \alpha, \beta), \quad v=v(\sigma ; \alpha, \beta)$,

where $\alpha$ and $\beta$ are real parameters (identifying the corresponding geodesic) while $\sigma$ is the arc length parameter.

(C) Select any function $\tau \rightarrow(\alpha(\tau), \beta(\tau))$ ( $\tau$ is a real variable) for which a transformation $(\sigma, \tau) \rightarrow(u$, v) given by

$u=u(\sigma ; \alpha(\tau), \beta(\tau)), \quad v=v(\sigma ; \alpha(\tau), \beta(\tau))$

is a diffeomorphism.

(D) One can show that in local coordinates $(\sigma, \tau)$ the metric of $\sum$ reads

$\mathrm{d} s^{2}=(\mathrm{d} \sigma)^{2}+2 g_{\sigma \tau}(\tau) \mathrm{d} \sigma \mathrm{d} \tau+g_{\tau \tau}(\sigma, \tau)(\mathrm{d} \tau)^{2}$.

Finally, we perform the following change of coordinates,

$x^{1}=\sigma+\int g_{\sigma \tau}(t) \mathrm{d} t$.

$x^{2}=\tau$,

leading to eq. (3).

(E) On calculating $b_{11}\left(x^{1}, x^{2}\right)$ we arrive at $f\left(x^{1}\right.$, $x^{2}$ ) given by eq. (5) while $S=S\left(x^{1}, x^{2}\right)=r_{1}\left(x^{1}, x^{2}\right)$ is a particular solution to eq. (1) with the same $f$.

The application of this algorithm to the unit sphere, cylinder and conus leads to the following results.

Sphere. We start with the parametric representation $r=[\cos u \cos v, \cos u \sin v$, $\sin u$ ]. Eq. (8) reads $u=\arcsin (\sin \sigma \cos \alpha)$,

$v=\beta-\arccos (\operatorname{tg} u \operatorname{tg} \alpha)$.

The transformation (9a) is given by

$x^{\prime}=\sigma+\int^{t} \beta^{\prime}(t) \sin \alpha(t) \mathrm{d} t$.

where the prime denotes ordinary differentiation and, finally, we have the associated coupling function 


$$
\begin{aligned}
& f\left(x^{1}, x^{2}\right) \\
& \quad=\alpha^{\prime}\left(x^{2}\right) \sin \left(x^{1}-\int \beta^{\prime}(t) \sin \alpha(t) \mathrm{d} t\right) \\
& +\beta^{\prime}\left(x^{2}\right) \cos \alpha\left(x^{2}\right) \\
& \quad \times \cos \left(x^{1}-\int \beta^{\prime}(t) \sin \alpha(t) \mathrm{d} t\right),
\end{aligned}
$$

and the corresponding solution to eq. (1) with $f$ given by (10) reads

$$
\begin{aligned}
& S=[\sin \gamma \sin \beta-\cos \gamma \cos \beta \sin \alpha, \\
& -\sin \gamma \cos \beta-\cos \gamma \sin \beta \sin \alpha, \\
& \cos \gamma \cos \alpha],
\end{aligned}
$$

where

$\alpha=\alpha\left(x^{2}\right), \quad \beta=\beta\left(x^{2}\right)$,

$\gamma=x^{1}-\int^{x^{2}} \beta^{\prime}(t) \sin \alpha(t) \mathrm{d} t$.

Cylinder. The corresponding parametric representation is given by $r=[\cos u, \sin u, v]$. Eq. (8) reads $u=\beta+\sigma \cos \alpha, \quad v=\sigma \sin \alpha$.

The transformation (9a) is given by

$x^{\prime}=\sigma+\int^{\tau} \beta^{\prime}(t) \cos \alpha(t) \mathrm{d} t$

As the associated coupling function we obtain

$$
\begin{gathered}
f\left(x^{1} ; x^{2}\right)=\sec ^{2}\left[\alpha\left(x^{2}\right)\right]\left[\beta^{\prime}\left(x^{2}\right) \sin \alpha\left(x^{2}\right)\right. \\
\left.-\alpha^{\prime}\left(x^{2}\right)\left(x^{1}-\int^{x^{2}} \beta^{\prime}(t) \cos \alpha(t) \mathrm{d} t\right)\right],
\end{gathered}
$$

and the corresponding solution to eq. (1) reads

$S=[-\sin \phi \cos \alpha, \cos \phi \cos \alpha, \sin \alpha]$,

where

$\alpha=\alpha\left(x^{2}\right)$,

$$
\begin{aligned}
\phi= & \beta\left(x^{1}\right)+\left(x^{2}-\int^{x^{2}} \beta^{\prime}(t) \cos \alpha(t) \mathrm{d} t\right) \\
& \times \cos \alpha\left(x^{2}\right) .
\end{aligned}
$$

It is worthwhile to point out that in the case under consideration, the cylinder, the coupling function (12) is a linear function in $x^{1}$ with general coefficients $a$ and $b$ depending on $x^{2}$, thus of the form $a\left(x^{2}\right) x^{1}+b\left(x^{2}\right)$. As we mentioned above this corresponds to the integrable case of eq. (2).

Cone. The corresponding parametric representation is given by $r=[u \sin \delta \cos v, u \sin \delta \sin v$, $u \cos \delta$ ], where $\delta$ is half the angle of the conus. Eq. (8) reads

$u=\sqrt{\sigma^{2}+\alpha^{2}}$,

$v=\operatorname{cosec} \delta[\operatorname{arctg}(\sigma / \alpha)+\beta]$.

The transformation $(9 a)$ is given by

$x^{1}=\sigma+\int^{\tau} \alpha(t) \beta^{\prime}(t) \mathrm{d} t$

As the associated coupling function we have

$$
\begin{aligned}
& f\left(x^{1}, x^{2}\right)=\operatorname{tg} \delta \alpha^{-2}\left(x^{2}\right) \\
& \quad \times\left[\alpha^{\prime}\left(x^{2}\right)-\beta^{\prime}\left(x^{2}\right) h\left(x^{1}, x^{2}\right)\right] \\
& \times\left[h^{2}\left(x^{1}, x^{2}\right)+\alpha^{2}\left(x^{2}\right)\right]^{3 / 2},
\end{aligned}
$$

and the corresponding solution to eq. (1) reads

$$
\begin{aligned}
S= & \sqrt{h^{2}\left(x^{1}, x^{2}\right)+\alpha^{2}\left(x^{2}\right)} \\
& \times\left[h\left(x^{1}, x^{2}\right) \sin \delta \cos \phi-\alpha\left(x^{2}\right) \sin \phi,\right. \\
& h\left(x^{1}, x^{2}\right) \sin \delta \sin \phi+\alpha\left(x^{2}\right) \cos \phi, \\
& \left.h\left(x^{1}, x^{2}\right) \cos \delta\right],
\end{aligned}
$$

where

$h\left(x^{1}, x^{2}\right)=x^{1}-\int^{x^{2}} \alpha(t) \beta^{\prime}(t) \mathrm{d} t$,

$\phi=\operatorname{cosec} \delta\left[\beta\left(x^{2}\right)+h\left(x^{1}, x^{2}\right) \operatorname{arctg} \alpha^{-1}\left(x^{2}\right)\right]$.

A few comments are in order. According to formula (6) it is always possible to calculate the corresponding $q$-solution from geometry (!). In all the discussed cases (sphere, cyclinder and conus) the surfaces are 
surfaces of rotation. It is not accidental. It is well known that in the general case of a pseudo-riemannian manifold any Killing vector field corresponds to some integral of motion of the equations for geodesics. The existence of such integrals simplifies greatly the implementing of the point (B) of the algorithm given above. In the case of ordinary surfaces in $E^{3}$ surfaces with rotational symmetry admit one Killing vector field in an obvious way. Indeed, one can find other $S$-solutions working with other surfaces of rotation (for instance with Beltrami's pseudosphere or the ellipsoid of rotation ).

Another interesting possibility is related to the wellknown local isometry between the surfaces of revolution and the so-called helicoids [12]. Investigations in this direction are in progress.

One of us (A. Sym) wishes to thank Professor Ruud Martini for his kind hospitality and moreover, both of us for his constant interest in the subject of the paper.

\section{References}

[1] R. Balakrishnan, J. Phys. C 15 (1982) L1305.

[2] M. Lakshmanan and R.K. Bullough, Phys. Lett. A 80 (1980) 287.

[3] R. Balakrishnan, Physica D 16 (1985) 405.

[4] D. Kaup, The nonintegrability of the generalized nonlinear Schrödinger equation, Clarkson University Preprint INS \#58 (February 1986)

[5] F. Calogero and A. Degasperis, Lett. Nuovo Cimento 22 (1978) 420.

[6] J. Ciesliński, P. Gragert and A. Sym, Exact solution to localized induction approximation eq. modelling smoke ring motion, to be published in Phys Rev, Lett.

[7] A. Sym, in: Geometric aspects of the Einstein equations and integrable systems, ed. R. Martini (Springer, Berlin, 1985) p. 154.

[8] L. Bianchi, Lezioni di geometria differenziale (Pisa. 1922)

[9] A. Sym, Lett. Nuovo Cimento 36 (1983) 307.

[10] A. Sym, Lett. Nuovo Cimento 41 (1984) 353

[11] M.P. Do Carmo, Differential geometry of curves and surfaces (Prentice Hall, Englewood Cliffs, 1976).

[12] V.F. Kagan, Principles of the theory of surfaces in the tensor presentation (Moscow, 1947) (in Russian). 